\title{
STABILIZATION OF THE DISTORTED CONFIGURATION OF THE EL2 DEFECT INDUCED BY THE FREE ELECTRON CAPTURE IN GaAsP*
}

\author{
J. Przybytek, M. Baj, T. Slupiński \\ Institute of Experimental Physics, Warsaw University \\ Hoża 69, 00-681 Warszawa, Poland
}

\begin{abstract}
Our results of optical absorption, electronic transport and deep level transient spectroscopy measurements performed on $n$-type $\mathrm{GaAs}_{1-x} \mathrm{P}_{x}$ $(x \approx 0.2)$ strongly suggest that using both an enlarged-gap material (compared to $\mathrm{GaAs}$ ) and hydrostatic pressure we can push down the acceptor level of the distorted configuration of the EL2 defect, (EL2* $)^{-10}$, sufficiently low into the gap that the distorted configuration of the EL2 defect, EL2*, becomes stabilized.
\end{abstract}

PACS numbers: 71.55.Eq

\section{Introduction}

It is well known that the EL2 defect in GaAs can be easily transformed (by means of illumination) to its distorted $\left(C_{3 v}\right)$ configuration. However, this configuration is unfavourable from the energetic point of view (it is metastable) and at sufficiently high temperatures $(\approx 40 \mathrm{~K}$ for $n$-type samples) the defect returns to its normal state (see e.g. [1] and references therein). Since the metastable defect $\left(\mathrm{EL} 2^{*}\right)$ can capture an additional electron (acceptor level of EL2* — see [2]), some energy can be gained in this process. If only this gain were sufficiently large, the distorted configuration of the EL2 defect could be stabilized. To maximize this gain we: (1) used a semiconducting material with an enlarged energy gap $\left(\mathrm{GaAs}_{1-x} \mathrm{P}_{x}, x \approx 0.2\right)$ and $(2)$ applied hydrostatic pressure, which additionally shifted the (EL2* $)^{-10}$ acceptor level down with respect to the conduction band states.

\section{Experiment and results}

The measurements of optical absorption were performed both: (1) at sufficiently high temperature $(\approx 80 \mathrm{~K})$ to enable an easy pressure-induced change of the defect configuration when it was energetically favourable and (2) at low temperatures $(\approx 10 \mathrm{~K})$, to avoid this transformation. At $T \approx 80 \mathrm{~K}$ we observed pressure-induced changes of the absorption such that at $p \approx 1 \mathrm{GPa}$ practically

*This work was partially supported by the State Committee for Scientific Research (Republic of Poland) grant No. 2 P302 01707. 
a)

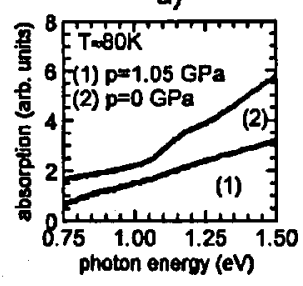

b)

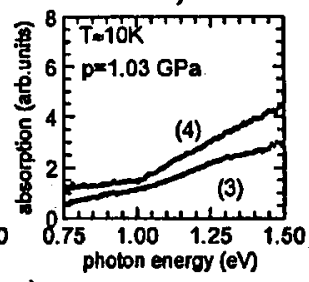

c)

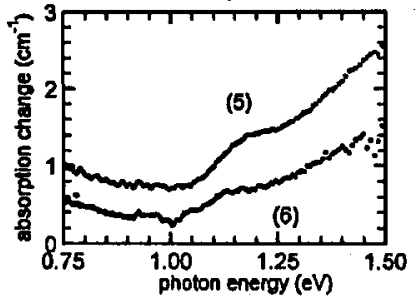

Fig. 1. (a) Pressure-induced reversible change in absorption under thermodynamic equilibrium conditions ( $T \approx 80 \mathrm{~K}$ ), (b) absorption spectra obtained after cooling down the sample to $10 \mathrm{~K}$ under pressure $1.03 \mathrm{GPa}$ in the dark - curve (3), and after optical recovery of the EL2 with near-band-edge light $(\approx 1.7 \mathrm{eV})$ - curve $(4),(\mathrm{c})$ for comparison: the changes in absorption induced by pressure - curve (5) and by light curve (6).

no characteristic EL2 absorption was seen (Fig. la, curve (1)). This means that the pressure-induced transformation of the EL2 to its distorted configuration took place in the dark. After a subsequent cooling down of the sample to $10 \mathrm{~K}$ at $p \approx$ $1 \mathrm{GPa}$, the EL2 defect remained frozen in its distorted configuration with no apparent characteristic absorption seen - Fig. 1b, curve (3). Then, still at $10 \mathrm{~K}$, by means of near-band-edge $(\approx 1.7 \mathrm{eV})$ illumination, we could recover the non-distorted configuration of EL2 with its well known absorption - Fig. 1b, curve (4). This efficient recovery is well known to take place in GaAs when the (EL2*) ${ }^{-10}$ level enters the gap under pressure [3].

We also performed Hall-effect measurements under pressure. To ensure thermodynamic equilibrium conditions the temperature was kept sufficiently high, at $T \approx 85 \mathrm{~K}$. When pressure was applied, the Hall free electron concentration decreased (see Fig. 2, curve for $85 \mathrm{~K}$ ) which correlated very well with the changes of the EL2 optical absorption measured at $80 \mathrm{~K}$ - i.e. also in thermodynamic equilibrium (Fig. 2, crosses). This experimental fact means that pressure-induced capture of free electrons is associated with the EL2 defect reconfiguration.

The Hall concentration data obtained at lower temperatures ( $T \leq 60 \mathrm{~K}$ ) showed the lack of thermodynamic equilibrium in that case. Applying pressure at high temperatures and cooling a sample down we could obtain various values of the free electron concentration (Fig. 2, curves for $40 \mathrm{~K}$ ) depending on the external conditions at which the sample was cooled. Slow kinetics of Hall concentration was also observed. Figure 3 presents the results of the following experiment: after cooling 


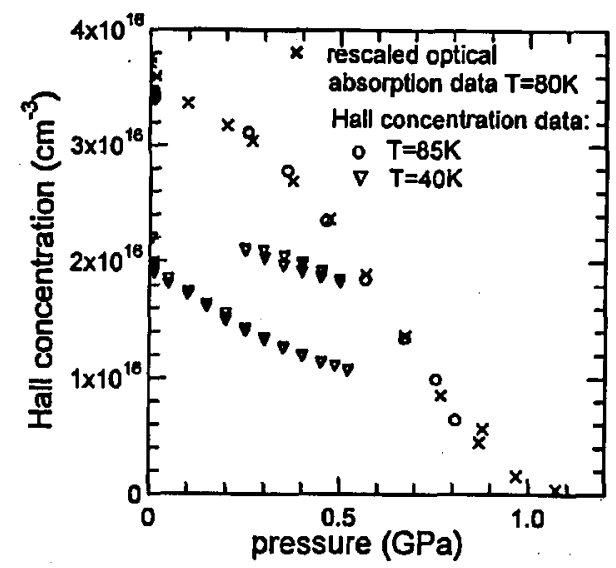

Fig. 2. The Hall-concentration data vs. pressure under thermodynamic equilibrium conditions (curve at $85 \mathrm{~K}$ ) and at $40 \mathrm{~K}$, when the concentration is frozen and reveals only slow kinetics. The crosses represent the pressure-induced quenching of the EL2-related absorption rescaled (for comparison) to fit the transport data.

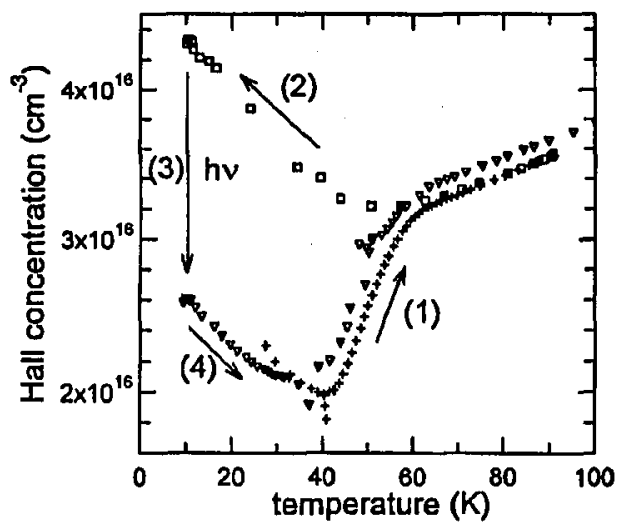

Fig. 3. A pressure-temperature cycle data: (1) heating up the sample after cooling down under pressure (ca. $0.8 \mathrm{GPa}),(2)$ cooling down without pressure and (4) heating up after illumination (3) with infrared LED. These data reveal that the both nonequilibrium states obtained by pressure (1) and illumination (4) unfreeze at the same temperature and in the same way.

the sample down to $28 \mathrm{~K}$ in the dark under pressure, we obtained a low value of the free electron concentration. We then released the pressure and, heating the sample, we measured a Hall concentration which revealed a step-like behaviour between $40 \mathrm{~K}$ and $60 \mathrm{~K}$ (Fig. 3, curve (1)). After that we cooled the sample down again (Fig. 3, curve (2)) and at low temperature we illuminated the sample with infrared light which caused a decrease in concentration (Fig. 3, arrow 3). During illumina- 
tion the EL2 defect was transformed, at least partially, to its EL2* distorted configuration which resulted in the capture of free electrons. We then heated the sample again (Figure 3, curve (4)): the concentration revealed almost the same step-like behaviour as for curve (1). This means that infrared light at low temperatures caused similar changes in the sample as pressure applied at high temperature.

Our deep level transient spectroscopy (DLTS) measurements fully correspond to the results given above. In a DLTS temperature scan, at $T \approx 100 \mathrm{~K}$ a peak emerged and rapidly grew in intensity (more than one order of magnitude) when pressure was applied. We attribute this signal to the (EL2*) ${ }^{-10}$ level, which arises from a spontaneous occupation of the EL2* distorted state. More details will be given elsewhere.

\section{Conclusions}

Very good correlation between the pressure dependence of the free electron concentration, intra-EL2 absorption and DLTS data strongly suggests that pressure- and alloying-induced lowering of the (EL2* $)^{-10}$ level with respect to the conduction band edge can cause a spontaneous transformation of the EL2 defect to its distorted EL2* $\left(C_{3 v}\right)$ configuration. We thus proved that it is possible to stabilize (under thermodynamic equilibrium conditions) the distorted configuration of the defect by capturing free electrons i.e. the distorted $C_{3 v}$ configuration becomes the fundamental one.

\section{Acknowledgments}

We are very much indebted to Dr. M.L. Sadowski for carefully reading the manuscript.

\section{References}

[1] M. Kamińska, Rev. Phys. Appl. 23, 793 (1988).

[2] M. Baj, P. Dreszer, A. Babiński, Phys. Rev. B 43, 2070 (1991).

[3] P. Dreszer, M. Baj, K. Kożuchowski, in: Proc. 16th Int. Conf. on Defects in Semicond., Bethlehem 1991, Eds. G. Davis, G.G. DeLeo, M. Stavola, Materials Science Forum, Vol. 83-87, Trans Tech Publications Ltd., Switzerland 1992, p. 871. 